VOL. $72(2005)$ [481-490]

\title{
RINGS HAVING ZERO-DIVISOR GRAPHS OF SMALL DIAMETER OR LARGE GIRTH
}

\author{
S.B. MULAY
}

\begin{abstract}
Let $R$ be a commutative ring possessing (non-zero) zero-divisors. There is a natural graph associated to the set of zero-divisors of $R$. In this article we present a characterisation of two types of $R$. Those for which the associated zero-divisor graph has diameter different from 3 and those $R$ for which the associated zero-divisor graph has girth other than 3 . Thus, in a sense, for a generic non-domain $R$ the associated zero-divisor graph has diameter 3 as well as girth 3 .
\end{abstract}

Let $R$ be a commutative ring with $1 \neq 0$ and let $Z(R)$ denote the set of non-zero zero-divisors of $R$. By the zero-divisor-graph of $R$ we mean the graph with vertices $Z(R)$ such that there is an (undirected) edge between vertices $x, y$ if and only if $x \neq y$ and $x y=0$ (see $[1,3,4])$. Since there is hardly any possibility of confusion, we allow $Z(R)$ to denote the zero-divisor graph of $R$. Following their introduction in [3], zerodivisor graphs have received a good deal of attention. For a more comprehensive list of references the reader is requested to refer to the bibliographies of $[1,2,4]$. Zero-divisor graphs are highly symmetric and structurally very special; for example, in [4] this author has investigated the structure of cycles, the graph-automorphism-group $\Gamma(R)$ and its explicit relationship with the ring-automorphism-group $\operatorname{Aut}(R)$. A sample consequence of interest is: if $\Gamma(R)$ is solvable, so is $\operatorname{Aut}(R)$. For this reason alone it is of interest to understand the nature of zero-divisor graphs. From the available evidence one is tempted to surmise that generic zero-divisor graphs may be completely classifiable (in some sense). It has been the experience that whenever one assumes $Z(R)$ to have some special feature, one can narrow down $R$ to a small class of rings. The present article provides an instance of this facet.

We tacitly assume that $R$ has at least 2 non-zero zero-divisors. By declaring the length of each edge to be $1, Z(R)$ becomes a metric space in which the distance between two vertices is, by definition, the length of a shortest path connecting them. The diameter of a metric space is the supremum (possibly $\infty$ ) of the distances between pairs of points of the space. With this structure, a zero-divisor graph is known to be a connected graph of diameter at most 3 (for example see [1] or [4, (1.2)]). The girth of a graph is the

Received 30th August, 2005

Copyright Clearance Centre, Inc. Serial-fee code: 0004-9727/05 \$A2.00+0.00. 
length of a shortest cycle (or equivalently the number of vertices of a least sided polygon) contained in the graph. If $Z(R)$ does not contain a cycle, then its girth is defined to be $\infty$. Obviously the girth of a graph is at least 3. The girth of $Z(R)$, for an arbitrary $R$, is known to be either infinite or 3 or 4 (see $[4,(1.4)]$ ).

In (1.2) of this article we present a characterisation of the rings $R$ for which the associated zero-divisor graph has diameter at most 2 . In (2.3) we identify those $R$ whose associated zero-divisor graph has girth exactly 4 . In (2.5) and (2.6) we determine the rings $R$ for which the associated zero-divisor graph has infinite girth. The graph-theoretic counterpart of this has already been dealt with in [4] where the zero-divisor graphs of infinite girth are completely determined. Except for one class of zero-divisor graphs of diameter 2, the nature of zero-divisor graphs having either small diameter or large girth is readily understood from the corresponding ring-theoretic characterisations.

By the total quotient ring of $R$ we mean the quotient ring $Q(R):=T^{-1} R$ where $T$ stands for the multiplicative subset of non zero-divisors of $R$. Since the canonical homomorphism from $R$ to $Q(R)$ is injective, $R$ is thought of as a sub-ring of $Q(R)$. As mentioned above, we shall tacitly assume $Z(R)$ to have at least two elements.

THEOREM 1.1. The diameter of $Z(Q(R))$ is the same as the diameter of $Z(R)$. The girth of $Z(R)$ is the same as the girth of $Z(Q(R))$.

PROOF: Observe that the diameter of $Z(Q(R))$ is 1 if and only if the diameter of $Z(R)$ is also 1 . Now suppose that the diameter of $Z(Q(R))$ is 2 . Then the diameter of $Z(R)$ is at least 2. Consider any $a, b \in Z(R)$ with $a \neq b$ and $a b \neq 0$. By our assumption about the diameter of $Z(Q(R)$, there is a $q \in Z(Q(R))$ such that $a \neq q \neq b$ and $a q=0=b q$. Let $q:=c / t$ with $c, t \in R$ such that $t$ is a non zero-divisor of $R$. Then $a c=0=b c$. It follows that $c$ is in $Z(R)$ and hence the distance between $a, b$ (when considered as vertices of the zero-divisor graph of $R$ ) is 2 . Conversely, assuming that the diameter of $Z(R)$ is 2 it is easy to see that the diameter of $Z(Q(R))$ must also be 2. In general, the diameter of any zero-divisor graph is at most 3 . Therefore we have established the first assertion.

Since $Z(R)$ is a sub-graph of $Z(Q(R))$, it is clear that the girth of $Z(R)$ is greater than or equal to the girth of $Z(Q(R))$. Earlier we have noted that the girth of any zerodivisor graph, when finite, is either 3 or 4 . Suppose $Z(Q(R))$ has girth 3 . Then there are distinct elements $q_{1}, q_{2}, q_{3}$ of $Z(Q(R))$ such that $q_{1} q_{2}=q_{2} q_{3}=q_{3} q_{1}=0$. For $i=1,2,3$ let $q_{i}:=a_{i} / t$ with $a_{i}, t \in R$ and where $t$ is a non zero-divisor of $R$. Then $a_{1}, a_{2}, a_{3}$ are distinct elements of $Z(R)$ and since $a_{1} a_{2}=a_{2} a_{3}=a_{3} a_{1}=0$, they form a triangle in the graph $Z(R)$. Thus $Z(R)$ also has girth 3 .

THEOREM 1.2. Assume that the diameter of $Z(R)$ is $\leqslant 2$. Then exactly one of the following holds.

(i) $Z(R) \cup\{0\}$ is a prime ideal of $R$, 
(ii) $R$ is a sub-ring of a product of two integral domains.

If (ii) holds for (a non-domain) $R$, then the diameter of $Z(R)$ is at most 2. If (i) holds for (a non-domain) $R$ and $R$ is Noetherian, then $Z(R)$ is of diameter at most 2 .

Proof: Suppose $Q(R)$ has two distinct maximal ideals $M_{1}$ and $M_{2}$. Let $x \in M_{1}$ and $y \in M_{2}$ be such that $x+y=1$. Then $x, y$ are in $Z(Q(R))$ and

$$
(0: x) \cap(0: y)=0
$$

(considered as ideals of $Q(R)$.) Since, by (1.1), the diameter of $Z(Q(R))$ is also 2, we must have $x y=0$. But $y=1-x$ and hence $x$ is an idempotent of $Q(R)$. It follows that $Q(R)$ is isomorphic to a product of two rings. Say $Q(R)=R_{1} \times R_{2}$. Suppose $Z\left(R_{1}\right)$ is non-empty. Let $a$ be an element of $Z\left(R_{1}\right)$. Then $(a, 1),(1,0)$ are elements of $Z(Q(R))$ such that the distance between them is at least 3 . This is impossible due to the assumption that $Z\left(Q(R)\right.$ has diameter 2 . Hence $Z\left(R_{1}\right)$ has to be empty. Symmetrically, $Z\left(R_{2}\right)$ must also be empty. Consequently $R_{1}, R_{2}$ are domains (in fact, fields) that is, assertion (ii) holds. It is clear that (i) holds if and only if $Q(R)$ has a unique maximal ideal.

If (ii) holds, then the diameter of $Z(R)$ is easily seen to be either 1 or 2 . If (i) holds and $R$ is Noetherian, then $P:=Z(R) \cup\{0\}$, being an associated prime of 0 , is of the form $(0: x)$ for some $x \in Z(R)$ and hence the diameter of $Z(R)$ is at most 2 .

REMARKS. 1. The diameter of $Z(R)$ is 0 if and only if $Z(R)$ is a singleton set if and only if $R$ is either $\mathbb{Z} / 4 \mathbb{Z}$ or $\mathbb{F}_{2}[X] / X^{2} \mathbb{F}_{2}[X]$ (or example, see $[4,(1.1)]$ ).

2. The diameter of $Z(R)$ is 1 (that is, $Z(R)$ is a complete graph) if and only if either $R$ is the product of the field of 2 elements with itself or (i) holds with the added property that $P^{2}=0$ (see [1]).

3. Let $A$ be a quasi-local factorial domain of (Krull) dimension at least 2. Let $m(A)$ denote the maximal ideal of $A$ and let $p(A)$ be a set of primes of $A$ such that for each height-one prime ideal $P$ of $A$ there is a unique $p \in p(A)$ with $P=p A$. Let $A[X]$ be the polynomial ring over $A$ in the set of indeterminates $X:=\left\{X_{p} \mid p \in p(A)\right\}$. Then $p(A)$ (and hence $X)$ is necessarily infinite. Let $J$ be the ideal of $A[X]$ generated by $\left\{p X_{p} \mid\right.$ $p \in p(A)\}$ and $I:=J+(X A[X])^{2}$. Define $R:=A[X] / I$ and $M:=(m(A)+X A[X]) / I$. Then $M$ is a maximal ideal of $R$ whose elements constitute the zero-divisors of $R$. Thus (i) holds for $R$. It is straightforward to verify that $A$ is (naturally) a sub-ring of $R$ and given two distinct members $p, q$ of $p(A)$ and an $r \in R$ with $p r=0=q r$ we must have $r=0$. Consequently, $Z(R)$ has diameter 3 .

LEMma 2.1. Assume that $Z(R)$ has girth 4. Then $R$ has at most one non-zero nilpotent. Furthermore, if $a$ is the non-zero nilpotent of $R$, then $(0: a)$ is a maximal ideal having $\mathbb{F}_{2}$ as its residue field.

Proof: Assume $R$ has non-zero nilpotents. Let $a$ be a non-zero nilpotent of $R$ such that $a^{2}=0$. If there are 4 distinct elements in the ring $R /(0: a)$, then there are 
3 distinct elements of $Z(R)$ of the form $x a, y a, z a$ (with $x, y, z \in R$ ) which constitute a 3 -cycle in $Z(R)$. Thus, in view of our hypothesis, it follows that $R /(0: a)$ is a ring of cardinality at most 3 . It is straightforward to verify that the zero-divisor graph of a ring of cardinality $<9$ has girth 3 . Hence $(0: a)$ has cardinality at least 4 . If $a R \neq\{0, a\}$, then for any $b \in a R \backslash\{0, a\}$ and any $x \in(0: a) \backslash\{0, a, b\}$ the elements $a, x, b$ of $Z(R)$ form a 3-cycle, contrary to our hypothesis. Therefore $a R=\{0, a\}$. It now follows that $(0: a)$ is a maximal ideal having the field of 2 elements as its residue field. If there is some non-zero $x \in(0: a)$ for which $(0: x) \cap(0: a)$ is not a subset of $\{0, a, x\}$, then for any $y \in(0: x) \cap(0: a) \backslash\{0, a, x\}$, elements $a, y, x$ form a 3-cycle. Hence $(0: x) \cap(0: a)$ is a subset of $\{0, a, x\}$ for all non-zero $x$ in $(0: a)$.

Let $y$ be a nilpotent of $R$ such that $y^{n+1}=0$ but $y^{n} \neq 0$ for a positive integer $n$. Clearly $n \leqslant 3$, otherwise, $y^{n}, y^{n-1}, y^{n-2}$ would be distinct elements of $Z(R)$ forming a 3-cycle. In other words $y^{4}=0$ for every nilpotent $y$ of $R$. Suppose there is a nilpotent $y$ in $R$ with $y^{3} \neq 0$. If $\left(0: y^{2}\right) \neq\left\{0, y^{2}, y^{3}\right\}$, then for any $z \in\left(0: y^{2}\right) \backslash\left\{0, y^{2}, y^{3}\right\}$ elements $y^{2}, z, y^{3}$ constitute a 3 -cycle. On the other hand, if $\left(0: y^{2}\right)=\left\{0, y^{2}, y^{3}\right\}$, then since $R /\left(0: y^{2}\right)$ has cardinality 2 by the above argument, $R$ would be a ring of cardinality at most 6 and hence $Z(R)$ can not possibly have girth 4 . Summarising, we must have $y^{3}=0$ for every nilpotent $y$ of $R$. Next suppose there is nilpotent $y$ of $R$ with $y^{2} \neq 0$. If $(0: y) \neq\left\{0, y^{2}\right\}$, then $y, x, y^{2}$ forms a 3-cycle of $Z(R)$ for any $x \in(0: y) \backslash\left\{0, y^{2}\right\}$. So $(0: y)=\left\{0, y^{2}\right\}$. Let $x$ be in $\left(0: y^{2}\right)$ but not in $(0: y)$. Then $x y$ is a non-zero element of $(0: y)$ and hence $x y=y^{2}$. Now $x-y$ being in $(0: y)$ it is either 0 or $y^{2}$. Thus $\left(0: y^{2}\right)$ is contained in the set $\left\{0, y, y^{2}, y+y^{2}\right\}$. Since $R$ must have cardinality at least 9 (for $Z(R)$ to have girth 4$)$ and $R /\left(0: y^{2}\right)$ has cardinality 2 , this is impossible. Therefore, we conclude that $y^{2}=0$ for each nilpotent $y$ of $R$.

Let $N(R)$ denote the nil-radical of $R$. Let $a, b$ be non-zero members of $N(R)$. If $a b \neq 0$, then elements $a, a+a b, a b$ form a triangle in $Z(R)$. This being impossible, $N(R)^{2}=0$. If $N(R) \neq a R$, then for any $c \in N(R) \backslash a R$ elements $a, a+c, b$ form a triangle of $Z(R)$. Hence we must have $N(R)=a R$. But we have already shown that $a R=\{0, a\}$. This establishes our assertion.

REMARK. Observe that if $R:=D \times \mathbb{Z} / 4 \mathbb{Z}$ where $D$ is an integral domain different from $\mathbb{F}_{2}$, then $R$ has a non-zero nilpotent and $Z(R)$ does have girth 4 .

LEMMA 2.2. Assume that the nil-radical of $R$ is zero. Then $Z(R)$ is complete bi-partite if and only if $R$ is a sub-ring of a product of 2 integral domains.

Proof: The 'if' part is straightforward. Suppose $Z(R)$ is complete bi-partite. Then $Z(R)$ has a partition $\left\{Z_{1}, Z_{2}\right\}$ where $Z_{1}=(0: x) \backslash\{0\}$ for all $x \in Z_{2}$ and $Z_{2}=(0: x) \backslash\{0\}$ for all $x \in Z_{1}$. Let $P_{1}:=Z_{1} \sqcup\{0\}$. Pick $y$ in $Z_{2}$. Now $P_{1}=(0: y)$. Assume $a, b \in R$ with $a b \in P_{1}$. Then $a b y=0$. If $b y=0$, then $b$ is in $P_{1}$. Assume $b y \neq 0$. Now $b y$ is in $Z(R)$ and $(0: y) \subseteq(0: b y)$. If $b y$ is in $Z_{1}$, then $0=(0: b y) \cap(0: y)$ which is absurd since $(0: b y) \cap(0: y)=(0: y)$. This forces by to be in $Z_{2}$. But then $(0: b y)=(0: y)$ 
and $a \in(0: b y)=(0: y)=P_{1}$. In other words, $P_{1}$ is a prime ideal of $R$. Likewise $P_{2}:=Z_{2} \sqcup\{0\}$ is also a prime ideal of $R$. Since $Z_{1} \cap Z_{2}$ is empty, $P_{1} \cap P_{2}=0$. It follows that $R$ is canonically isomorphic to a sub-ring of the product of integral domains $R / P_{1}$ and $R / P_{2}$.

THEOREM 2.3. Assume that $Z(R)$ has finite girth. Then the girth of $Z(R)$ is 4 if and only if one of the following holds.

(i) $R$ is a sub-ring of a product of two integral domains.

(ii) $R$ is isomorphic to $D \times S$ where $D$ is an integral domain and $S$ is either $\mathbb{Z} / 4 \mathbb{Z}$ or $\mathbb{F}_{2}[X] / X^{2} \mathbb{F}_{2}[X]$.

Proof: The proof is divided in two cases, namely the case where the nil-radical $N(R)$ is zero and the case where $N(R)$ is non-zero. Since $R$ must have at least 9 elements for $Z(R)$ to have girth 4 , henceforth we tacitly assume that the cardinality of $R$ is at least 9.

First assume that $N(R) \neq 0$. Then, from $(2.1)$ it follows that $N(R)=\{0, a\}$ and the cardinality of $(0: a)$ is at least 5 . Let $x$ be a non-nilpotent in $(0: a)$. If $(0: x) \cap(0: a)$ contains a non-nilpotent $y$, then $x, a, y$ form a triangle in $Z(R)$. Hence $(0: x) \cap(0: a)=N(R)$ for every non-nilpotent $x$ in $(0: a)$. If every zero-divisor of $R$ is in $(0: a)$, then $(0: x)=N(R)$ for all non-nilpotents of $Z(R)$ and hence $Z(R)$ has infinite girth contrary to our assumption. Thus $Z(R) \backslash(0: a)$ must be non-empty. Pick $y \in Z(R)$ such that $y$ is not in $(0: a)$. Clearly, $(0: y) \neq 0$. If $(0: y) \cap(0: a)$ is a subset of $N(R)$, then since $a y \neq 0$, we have $(0: y) \cap(0: a)=0$ and hence $R$ is isomorphic to $\mathbb{F}_{2} \times R /(0: y)$ (where the first factor is the field of 2 elements). But the zero-divisor graph of such a product has girth either $\infty$ or 3 . Thus it must be possible to choose a non-nilpotent $x$ in $(0: y) \cap(0: a)$. Consider the set $C:=\{z y \mid z \in(0: a)\}$. If $C$ has a member $b$ not in $\{0, x, a\}$, then $x, b, a$ form a triangle of $Z(R)$ contrary to our hypothesis. Therefore, $C$ is contained in $\{0, x, a\}$. Using the fact that $R /(0: a)$ is the field of two elements, we conclude that $y-1$ is in $(0: a)$ and $y R$ is a subset of $\{0, x, a, y, y+x, y+a\}$. Now $(y-1) a=0$ implies that $a$ is in $y R$. So $0, y, a, y+a$ are 4 distinct elements of $y R$. Clearly, from our choice of $x, y$ it follows that $y+x+a$ can not be in the set $\{0, x, a, y, y+x, y+a\}$. Hence $y R=\{0, y, a, y+a\}$. But then $y^{2}$ must belong to $\{y, y+a=y(1+a)\}$ (observe that $1+a$ is a unit of $R$ ). Consequently, $y^{2} R=y R$ has exactly 4 elements, $(0: y) \cap y R=0$ and $(1-y)$ is in $(0: y)$. Thus $R$ is isomorphic to $R / y R \times R /(0: y)$ where $R /(0: y)$ is a ring of cardinality 4 containing a non-zero nilpotent (namely, the image of $a$ ). In other words (ii) holds.

Finally consider the case where $N(R)=0$. Let $x$ be in $Z(R)$. Suppose, if possible, that $(0: x)$ has exactly 2 elements $\{0, y\}$. Then $y R=\{0, y\}$ and hence $(0: y)$ is a maximal ideal having the residue field of 2 elements. Also, since $y^{2} \neq 0$, we must have $y^{2}=y$ and $y R \cap(0: y)=0$. Hence $R$ is isomorphic to $\mathbb{F}_{2} \times R / y R$. But such a ring has girth either $\infty$ or 3 . Thus for each $x$ in $Z(R)$, the cardinality of $(0: x)$ is at least 3 (in 
the terminology of [4], the graph $Z(R)$ has no 'ends'). Now it follows from $[4,(2.2)]$ (see Remark 2. following the assertion $[4,(2.2)])$ that $Z(R)$ is a bi-partite graph. In view of the Lemma (2.2) of this article we see that (i) holds.

Conversely, if either of (i) or (ii) holds (with $Z(R)$ being non-empty of finite girth) then it is easy to verify that the girth of $Z(R)$ is exactly 4 .

Lemma 2.4. Assume that $R$ has at least 10 elements. Let $N(R)$ be the nil-radical of $R$ and assume that $N(R) \neq 0$. Then the following are equivalent.

(i) $Z(R)$ has infinite girth.

(ii) $N(R)=\{0, y\}$ and $(0: x)=N(R)$ for all $x \in Z(R)$.

(iii) $N(R)$ has cardinality 2 and it is a prime ideal of $R$.

Proof: The equivalence of (i) and (ii) follows from [4, (2.1)]. Assertion (iii) follows from (ii) in a straightforward manner. Suppose (iii) holds. Then $N(R)=\{0, y\}$ for some non-zero $y$ in $R$. Let $x \in Z(R)$ be distinct from $y$. Then $x$ is not in $N(R)$ and $(0: x) \neq 0$. Consider $0 \neq w \in(0: x)$. Since $0=x w \in N(R)$ and $N(R)$ is prime, we must have $w \in N(R)$ and hence $w=y$. Hence (ii) holds.

Definition: Let $B$ be a ring such that its nil-radical $N(B)$ is a prime ideal of cardinality 2 and let $B[X]$ be the polynomial ring in a non-empty set of indeterminates $X$ over $B$. Let $I$ be an ideal of $B[X]$ such that

1. $I \cap B=0$,

2. $N(B) B[X] \cdot X B[X] \subseteq I \subseteq 2 B[X]+N(B) B[X]+X B[X]$ and

3. $P(B, X, I):=N(B) B[X]+I$ is a prime ideal of $B[X]$.

Then, by $\rho(B, X, I)$ we mean the ring $B[X] / I$.

THEOREM 2.5. Assume that $R$ has at least 10 elements. Let $N(R)$ be the nilradical of $R$ and assume that $N(R) \neq 0$. Then $Z(R)$ has infinite girth if and only if one of the following holds.

(i) $\quad R=\rho(B, X, I)$ where $B=\mathbb{Z}[w] /\left(w^{2}, 2 w\right) \mathbb{Z}[w]$ for an indeterminate $w$ over $\mathbb{Z}$.

(ii) $\quad R=\rho(B, X, I)$ where $B=\mathbb{F}_{2}[w] / w^{2} \mathbb{F}_{2}[w]$ for an indeterminate $w$ over $\mathbb{F}_{2}$ and where $I$ is such that $P(B, X, I) \neq N(B) B[X]+X B[X]$.

(iii) $R=\rho(B, X, I)$ where $B=\mathbb{Z} / 4 \mathbb{Z}$ and $I$ is such that $P(B, X, I)$ $\neq N(B) B[X]+X B[X]$.

Moreover, such a ring is necessarily infinite.

Proof: Our argument will tacitly employ Lemma (2.4). At the outset we show that under our assumptions the characteristic of $R$ is either 0 or 2 or 4 . Observe that a sub-ring of $R$ does not contain $y$ if and only if it is an integral domain. On the other hand, if a sub-ring $S \subseteq R$ contains $y$, then $N(S)=N(R) \cap S$ is a prime ideal and for any $a, b \in Z(S)$ we have $a b=0$ if and only if either $a=y$ or $b=y$. Also, it follows that 
$(0: y) \cap S$ is a maximal ideal of $S$ with residue field $\mathbb{F}_{2}$. In particular, the characteristic of $R$ is an even integer. Let $2 n$ denote the characteristic of $R$. Suppose $n$ is neither 0 nor 1. Then $y$ is in the prime sub-ring $\pi$ of $R$. Since the zero-divisors of $\pi$ have to be contained in the maximal ideal $(0: y) \cap \pi$, the ring $\pi$ is a local ring that is, $n$ is a power of 2. But the nil-radical of $\pi$ has exactly two elements. Hence $n=2$. It is plain to see that the rings of the form mentioned in (i), (ii), (iii) above have characteristics 0,2 and 4 respectively.

Suppose $R$ satisfies any one of (i), (ii) and (iii). To simplify the notation set $P:=P(B, X, I)$. In the first two cases let $t$ be the canonical image of $w$ in $B$ and in the third case let $t=2$. Note that $t^{2}=2 t=0$ and $N(B)=\{0, t\}$. We claim that $R$ has to be infinite. This is evident in the case of (i) since $\mathbb{Z}$ is indeed the prime sub-ring of $R$. In the remaining two cases there is an $x$ in $X$ which is not in $P=t B[X]+I$ Consider the sub-ring $A$ of $B[X]$ obtained by adjoining $x$ to the prime sub-ring. Then $A$ is a polynomial ring in one variable over the prime sub-ring and $I \cap A \subseteq P \cap A$. If $A$ has characteristic 2 then $P \cap A \subseteq x A$ and consequently $P \cap A=0$. Thus $A$ is (naturally) an infinite sub-ring of $R$. If $A$ has characteristic 4, then $P \cap A=2 A$ and hence $A /(I \cap A)$ is necessarily an infinite sub-ring of $R$. Let $y$ denote the canonical image of $t$ in $R$. Then $y$ is a non-zero nilpotent of $R$. Let $f \in B[X]$ be in the radical of $I$. Clearly $f$ has to be in $P$. Hence $y R=P / I=N(R)$ is a prime ideal of $R$. It is easy to verify that $N(R)=\{0, y\}$. By Lemma (2.4), $Z(R)$ must have infinite girth.

Conversely, suppose $R$ has at least 10 elements, $N(R) \neq 0$ and $Z(R)$ has infinite girth. In view of Lemma $(2.4)$ if we let $N(R):=\{0, y\}$, then $N(R)$ is a prime ideal and $(0: y)$ is a maximal ideal with residue field $\mathbb{F}_{2}$. We have already established that the characteristic of $R$ has to be one of $0,2,4$. Our assumption about the cardinality of $R$ ensures that the ideal $(0: y)$ is distinct from $N(R)$. Let $\pi$ denote the prime sub-ring of $R$. Then $B:=\pi[y]$ is (isomorphic to) exactly one of the rings appearing in (i), (ii), (iii) above. Clearly, $N(B)=N(R)$ and since for each $r \in R$ either $r$ or $r+1$ is in $(0: y)$, the ring $R$ is obtained by adjoining the elements of $(0: y)$ to $B$. Observe that $(0: y) \cap B$ is a maximal ideal of $B$ which equals $J:=2 B+N(B)$ and has $\mathbb{F}_{2}$ as its residue field. Now the $B$-module $(0: y) / J$ is in fact a vector-space over $\mathbb{F}_{2}=B / J$. Let $T \subset(0: y)$ be such that $T / J$ is an $\mathbb{F}_{2}$-basis of $(0: y) / J$ (we allow $T$ to be the empty set). Then $R=B[T]$. Let $X$ be a set of indeterminates over $B$ equipped with a bijection $s: X \rightarrow T \cup\{0\}$. Let $\sigma: B[X] \rightarrow R$ be the unique homomorphism of $B$-algebras which restricts to $s$ on the set $X$. Then $\sigma$ is surjective. Let $I$ denote the kernel of $\sigma$. Obviously $I \cap B=0$ and $I$ contains $y x$ for all $x \in X$. If $f:=b-g \in I$ with $b \in B$ and $g \in X B[X]$, then $b=\sigma(b)=\sigma(g)$ and $\sigma(g) \in(0: y)$ imply that $b$ is in $J$. Consequently, $I$ is contained in $2 B[X]+N(B) B[X]+X B[X]$. Finally, since $\sigma(N(B))=N(R)$ is a prime ideal of $R$, its inverse image $N(B) B[X]+I$ is also a prime ideal of $B[X]$.

REMARKs. 1 . If $R$ has at least 10 members, $N(R)=0$ and $Z(R)$ is non-empty, then 
$Z(R)$ has infinite girth if and only if $R$ is a product of a domain $D$ and $\mathbb{F}_{2}$. This assertion follows from the remark at the end of $[4,(2.1)]$ (for infinite rings $R$ see [1, Theorem 2.5]). In fact, the above Theorem, in conjunction with $[4,(2.1)]$ provides a complete (without any cardinality restrictions) characterisation of those non domains $R$ for which $Z(R)$ has infinite girth (that is, $V(R)=\emptyset$ in the notation used in [4]).

2. Note that in the above proof it is not essential for us to choose $T$ the way we have chosen it, that is, we did not make any particular use of the fact that $T / J$ is a vector-space basis. On the other hand, it is natural to try to get hold of a 'smallest possible' set $T$ with $R=B[T]$.

Definition: If $B$ is a ring and $M$ is a $B$-module, then by $B(+) M$ we denote the ring obtained by idealising $M$ (as defined in [5] ).

THEOREM 2.6. Assume $R$ satisfies the following. As above, $N(R)$ denotes the nil-radical of $R$.

(i) $R$ has at least 5 elements.

(ii) There is $y \in Z(R)$ such that $N(R)=\{0, y\}$ and $(0: x)=N(R)$ for all $x \in Z(R)$.

Then the characteristic of $R$ is not 4 if and only if $R$ has a sub-domain $A$ such that $A[y] \cong A(+) \mathbb{F}_{2}$ and for each $r \in R$ there exists a non-zero $a \in A$ (depending on $r$ ) with $a r \in A[y]$. Moreover, if $r$ is not in $N(R)$ then $a r \neq 0$. (David $F$. Anderson (in a private communication) asked whether a non-reduced, non-domain $R$ with $Z(R)$ having infinite girth is of the form $D(+) \mathbb{F}_{2}$ for some domain $D$. The above theorem constitutes our response to his question.)

PROOF: From the argument at the beginning of the proof of (2.5) it follows that $R$ has characteristic 0 or 2 or 4 . If a ring contains a sub-ring of type $D(+) \mathbb{F}_{2}$ with $D$ a domain, then obviously the characteristic can not equal 4 . Henceforth, assume that the characteristic of $R$ is either 0 or 2 . We proceed to show that $R$ contains an infinite integral sub-domain. This is evident if $R$ is of characteristic 0 . Suppose $R$ has characteristic 2 . Condition (i) ensures that $Z(R) \backslash\{y\}$ is non-empty. Let $x$ be an element of $Z(R) \backslash\{y\}$ and $S:=\mathbb{F}_{2}[x]$. Note that $Z(S)$ is contained in the single maximal ideal $P:=(0: y) \cap S$ of $S$. Since $x S \subseteq P$, we must have $P=x S$. It is easy to see that $y$ is not in $x R$ and hence $y$ is not in $S$. Thus $S$ is an infinite integral sub-domain of $R$ (in fact a polynomial ring over $\mathbb{F}_{2}$ ). Let $A$ denote a maximal sub-ring of $R$ not containing $y$. Existence of such a sub-ring can be seen in a straightforward manner. As a consequence of the above argument $A$ has to be an infinite integral domain. Since $(0: y) \cap A$ is a maximal ideal of $A$ with residue field $\mathbb{F}_{2}$, it follows that $A$ is not a field. For $r \in R$ define

$$
I(r, A):=\{a \in A \mid \text { ar } \in A[y]\} .
$$

Clearly $I(r, A)$ is an ideal of $A$ and $I(r+b, A)=I(r, A)$ for all $b \in A$.

Suppose there is an $x$ in $R$ with $I(x, A)=0$. Then $I(b x, A)=0$ for all non-zero $b \in A$. Replacing $x$ by $x+1$ if needed, we may assume that $x$ is a member of $(0: y)$. Obviously 
$x$ is not in $N(R)$ and hence $x R \cap N(R)=0$. Consider the $A$-algebra homomorphism $h$ of the polynomial domain $A[X]$ onto $A[x]$ which maps the indeterminate $X$ to $x$. Let $J(x)$ denote the kernel of $h$. Our choice of $A$ ensures that $y \in A[x]$. Thus $J(x)$ is not a radical ideal of $A[X]$. In particular, $J(x) \neq 0$. Let $f \in J(x)$ be a non-zero polynomial of least degree. Let $f:=a_{0} X^{d}+\cdots+a_{d}$ where $d \geqslant 2$ is the degree of $f$ and $a_{0} \neq 0$. Replacing $x$ by $a_{0} x$ if needed, we may assume $a_{0}=1$ that is, $x$ is integral over $A$. The minimality of $d$ allows us to conclude that $J(x)=f A[X]$. More generally, we observe that $J(b x)=b^{d} f(X / b) A[X]$ for all non-zero $b \in A$. Consequently, for each non-zero $b \in A$, the ring $A[b x]$ contains $y$ and it is a free $A$ - sub-module of $A[x]$ with basis $\left\{1, b x, \cdots,(b x)^{d-1}\right\}$. Now, $A$ is an integral domain which is not a field and hence the intersection of all non-zero principal ideals of $A$ is necessarily zero. Thus the intersection of all the sub-modules $A[b x]$ of $A[x]$, as $b$ ranges over non-zero elements of $A$ is exactly $A$. This is absurd since $y$ is certainly not in $A$. Therefore we must have $I(r, A) \neq 0$ for all $r \in R$. It is easy to see that $A[y] \cong A(+) \mathbb{F}_{2}$.

Remarks. 1. The above theorem allows us to think of $R$ as a "blow-up" of $A(+) \mathbb{F}_{2}$.

2. A ring $R$ is of the form $S(+) M$ for some non-zero $S$-module $M$ if and only if $R$ has a non-zero ideal $N$ and a derivation $\delta: R \rightarrow N$ such that $N^{2}=0, N \cap \operatorname{Ker}(\delta)=0$ and the set-theoretic map $R \rightarrow R / N \oplus N$ sending $r \in R$ to $\sigma(r) \oplus \delta(r)$ (here $\sigma$ is the canonical map) is surjective. Now any derivation of $R$ is identically 0 on the prime subring of $R$. Suppose $R$ satisfies the conditions of the above Theorem and the characteristic of $R$ is 4 . Then $N(R)=\{0,2\}$ and it is the only non-zero ideal of $R$ whose square is zero. Since $N(R)$ is contained in the kernel of every derivation of $R$, it follows that $R$ is not of the form $S(+) M$ with $M \neq 0$.

3. Consider the 3-variable polynomial ring $B:=\mathbb{F}_{2}\left[X_{1}, X_{2}, X_{3}\right]$ with ideal $I$ generated by $\left\{X_{1} f, X_{2} f, X_{3} f\right\}$ where $f:=X_{1} X_{3}+X_{2}^{2}$. Let $R:=B / I$ and let $u, v, w, y$ denote the canonical images of $X_{1}, X_{2}, X_{3}, f$ (respectively) in $R$. It is easy to see that $R$ satisfies the hypotheses of the above Theorem. We leave it to the reader to verify that for $A$ (as in the conclusion of the Theorem) we may take the sub-ring $\mathbb{F}_{2}\left[u, v, w^{2}, v w\right]$. Further, it is simple to check that $\delta(y)=0$ for any derivation $\delta: R \rightarrow\{0, y\}$. Hence $R$ is not of the form $S(+) M$ with $M \neq 0$. One can construct a similar example in characteristic 0 .

\section{REFERENCES}

[1] D.F. Anderson and P.S. Livingston, 'The zero-divisor graph of a commutative ring', $J$. Algebra 217 (1999), 434-447.

[2] D.F. Anderson, R. Levy and J. Shapiro, 'Zero-divisor graphs, von Neumann regular rings and Boolean algebras', J. Pure Appl. Algebra 180 (2003), 221-241.

[3] I. Beck, 'Coloring of commutative rings', J. Algebra 116 (1988), 208-226.

[4] S.B. Mulay, 'Cycles and symmetries of zero-divisors', Comm, Algebra 30 (2002), 3533-3558.

[5] M. Nagata, Local rings (Krieger Publishing Company, Huntington, N.Y., 1975). 
Department of Mathematics The University of Tennessee Knoxville, TN 37996 United States of America e-mail: mulay@math.utk.edu 